\title{
Tropical Diseases in Saudi Arabia
}

\author{
Alaa E Ahmed* \\ Medical Research Center, Jazan University, Saudi Arabia
}

Received: May 10, 2015; Accepted: May 14, 2015; Published: May 22, 2015

*Corresponding author: Alaa E Ahmed, Medical Research Center, Jazan University, Saudi Arabia, E-mail: alaa-ahmed@msn.com

\begin{abstract}
The Kingdom of Saudi Arabia, with its vast geographical area, and its location at the boundary of the equatorial, has seen recently several outbreaks of either tropical or Neglected Tropical Diseases (NTD). Being the center of the Islamic world, with millions of pilgrimages visiting the holy sites in Saudi Arabia in the annual sessions of pilgrimage and Umrah, puts the world health at risk of wide and uncontrolled epidemics with its close boundaries with both Yemen and Egypt, which are the most countries in the Middle East region harboring NTD's, puts the Kingdom at an additional risk together with the greenhouse effect of global warming and climate changes. The current status of the NTDs in Saudi Arabia is currently unknown due to several factors of which, poor reporting, lack of proper health workers training and surveillance efforts, being the most important factors. This mini-review will summarize the types of NTD's diseases and current status in Saudi Arabia.
\end{abstract}

\section{Introduction}

Diseases unique to the tropical and subtropical regions (tropical diseases) are defined as Tropical medicine or International Medicine. More recently the term Neglected Tropical Diseases (NTD) has been introduced through the initiative of the World Health Organization [1]. NTD's (about 17 diseases) are mostly infectious in nature and requires a vector for their propagation, and are prevalent in the tropical and subtropical regions, they were endemic even in countries with cold climates; however with improvement with sanitation, heath education and personal hygiene [2], in addition to the cold session, which in part force the vector to hibernation, those diseases have been eliminated or controlled.

NTD's has a heavy burden on public health as they promote poverty, reduced population production, child health, pregnancy and health cost. Each NTD has its own unique etiological features, geographic distribution and public health burden [3,4].

The most common vector for NTD's transmission is insects (mosquitoes and flies), which may carry a parasite, bacterium or virus that is infectious to humans and animals and may act as a biological reservoir for the pathogen multiplication and/ or incubator. In common, diseases transmission is caused by an insect "bite", through subcutaneous blood exchange [5].

Recent climate changes, also known as greenhouse effect, in addition to, deforestation, rising immigration and increased international air travel and other tourism to tropical regions has contributed to an increased incidence of NTD's, which is can cause high incidence of morbidity and mortality $[6,7]$.

Thelocalization of tropical diseases is in poor, underdeveloped countries spanning the tropics, mainly in Africa, Asia, Central America and South America, has led several international efforts to combat those array of devastating diseases. The financial burdens of those diseases are substantial $[2,8]$.

While much of the current international efforts have been focused on four diseases, namely Tuberculosis, Malaria, HIV and Viral hepatitis, much less efforts or research has been geared toward other NTD's. Currently, more than a billion individual is infected globally with NTD's, including 500 million children according to the latest WHO estimate [8].

The Kingdom of Saudi Arabia with its vast borders, and especially its southern borders with close proximity to the African horn and South East Asia, in addition to its subtropical climates, puts the kingdom at risk for an epidemic or endemic episodes of NTD's.

According to recent estimates, the kingdom ranks the highest second country in the Middle East North Africa (MENA) region for Leishmaniasis (L. major) infection, with more than 4,000 reported cases; and the third country for Hookworm infection in the region, with 0.4 million reported cases. Lymphatic filariasis also has been reported in Saudi Arabia [9]. Accurate estimates of the NTD's cases in Saudi Arabia are often unavailable, due to health system infrastructure, inefficient reporting system, beside other factors [9].

\section{Onchocerciasis}

It is the world's second leading infectious cause of blindness. It is caused by Onchocerca parasitic worm. It is mode of transmission is through black fly. Infection usually spread very rapidly in patients leading to an immune response and destruction of tissue, especially the eye. The prevalence in the Kingdom is currently unknown. Another form of Onchocerciasis, known as Aswad with severe dermatitis and edema is well documented in Yemen; however information about its prevalence in the kingdom is also lacking $[10,11]$.

\section{Leishmaniasis}

Both cutaneous and visceral Leishmaniasis occurs in Saudi 
Arabia. The cutaneous type is common throughout the kingdom especially in Eastern and Central provinces [12]. The visceral type which is more virulent is confined to the South and Southwest provinces. Dogs have been identified as the animal reservoir; however, accurate information on the national prevalence of the disease is not known [13]. Several studies have indicated the presence of several hot areas of the diseases in different parts of the country $[12,13]$.

\section{Toxoplasmosis}

(Cat disease) is another important protozoan infection thought to be present throughout the kingdom, with unknown national prevalence [14]. Recent study reported that the seropositivity of 487 pregnant women for Toxoplasma gondii infection as high as $38.8 \%$ in the south western region of Saudi Arabia [14]. Toxoplasmosis infection was also reported in other regions of the country [15-17]. Toxoplasmosis infection is also reported to be widely spread between animal species in Riyadh region [18]. Toxoplasmosis is currently considered as a global health threat as it infects $40-50 \%$ of the world population [19].

\section{Brucellosis}

It is a major health concern in Saudi Arabia. Its high prevalence has been largely attributed to the social norms of the nomadic population who live in close proximity with livestock and consume raw milk, additionally, associated breakdowns in veterinary health control systems and unrestricted animal transportation through open borders have promoted the reemergence of brucellosis in the Kingdom [20,21]. National prevalence of the infection spread is currently unknown [9].

\section{Dengue Virus (DENV)}

A mosquito's transmitted disease is endemic in the western and southern regions of the Kingdom, with outbreaks occurring in 2006 and 2008. Recent studies indicated the persistent endemic in the southern and western regions [22,23]. Three strains of the 4 DENV strains were reported in the region and also in neighboring Yemen [24]. Climate changes, increasing work force from DENV endemic areas, plus increased urbanization will eventually leads to increased risks of future outbreaks.

\section{Rift Valley fever (RVF)}

It is also a mosquito's transmitted disease caused an outbreak in 2000 in the Jazan region and other parts of the Kingdom [25]. This was the first recorded outbreak outside of Africa, affecting several thousand animals and resulting in approximately 1,000 positive cases and several hundred deaths [26]. Public health measure untaken to combat the spread of the disease seems to be working and should be maintained [27].

\section{Alkhurma Hemorrhagic Fever (AHFV)}

It is caused by a tick-borne flavivirus, has also emerged on the Kingdom in the Najran region in 1996. The knowledge gaps about those viruses are still wide [28]. Recent reports indicate that the epidemiology of the diseases is spreading among the population and is moving outside its geographic areas [29].

\section{Trypanosomiasis}

Prevalence of African Trypanosomiasis infection in humans, which is a parasitic disease, caused by protozoa called trypanosomes and transmitted by the tsetse fly, is unknown in the Kingdom [9]. Increased prevalence in animals and livestock was noted in many animal species including camels, sheep and cattle's. Jazan region showed the highest level of infection rate of camels of $40 \%$ with new species identified for the first time in the country [30].

\section{Lymphatic filariasis}

It is well documented in Yemen, is a parasitic disease caused by parasitic filarial worms transmitted by mosquitoes. Loa loa is another filarial parasite transmitted by the deer fly. However, the prevalence in the Kingdom is unknown.

\section{Malaria}

It has been documented as a major health problem in several regions of the Kingdom. Malaria is caused by a Protozoan parasites transmitted by female Anopheles mosquitoes. The population at risk of malaria in the Kingdom is estimated to be 1.4 million and malaria epidemics frequently occur after heavy rains sessions. On other hand, the existence of malaria in Southwestern region of the Kingdom is greatly affected by the active and continues importation of cases and vectors from Yemen. Malaria vector control has been the focal aim for the health authorities, Agriculture and local authorities. With the emergence of drug resistant strains, the efforts should be maximized.

Both intestinal and urinary Schistosomiasis has been prevalent in Saudi Arabia. Schistosomiasis is a parasitic disease caused by several species of flatworm. Despite rigorous combating efforts of the diseases in the kingdom, the current prevalence and hot areas of the disease is unknown.

\section{Tuberculosis (TB)}

It is a bacterial infection of the lungs or other tissues, which is highly prevalent in the world, with mortality rate of over $50 \%$ if untreated. It is a communicable disease, transmitted by aerosol expectorant from a cough, sneeze, speak, kiss, or spit. Over one-third of the world's population has been infected by the TB bacterium. The situation of TB in Saudi Arabia is unique. The country has interesting and special population dynamics. There are up to ten million expatriates mainly from endemic regions, in South and South East Asia and over two million pilgrims visiting the holy cities located in the western region of the Kingdom each year, with the majority of pilgrims coming from endemic areas. The current status of TB in the kingdom is unknown, especially the new drug resistant strains.

While medical therapeutics and vaccines are the first line of defense against tropical diseases, and despite the success of vaccine development and manufacturing globally in combating many diseases and alleviating the socioeconomic burden of those diseases on public health, the need for more vaccine research and development in tropical medicine is at its beak currently, as evident by the race to develop an HIV, Hepatitis C or Malaria vaccines are underway. 
While vaccine development is a highly complex and lengthy process as it may take from 10 to 12 years, and involve multiple stages. The first stage involves the isolation of the pathogen including all of its variants, and identifies the antigens. The second stage is a preclinical stage which begins with antigen characterization and purification to develop appropriate vaccine candidates. Animal studies are used to determine the immunogenicity and safety of the candidate's vaccine.

Another area of concern is the lack of rapid diagnostic devices to screen large number of patients rapidly and cost effectively with superior analytical and clinical sensitivity and specificity in case of sudden outbreaks.

Tropical disease research is lacking in the Kingdom and the region in general, where approximately more than $90 \%$ of human and veterinary medicine or vaccines are imported, which poses a serious public health burden and threat. Depending on foreign suppliers is plagued by many risks of sudden outbreak, faulty manufacturing, and surge in demands globally or market shift. The Kingdom is in perfect position to lead international efforts on tropical diseases research.

\section{References}

1. Hotez PJ, Fenwick A, Savioli L, Molyneux DH. Rescuing the "bottom billion" through neglected tropical disease control. Lancet. 2009; 373(9674): 1570-1574. doi: 10.1016/S0140-6736(09)60233-6.

2. World Health Organization. Working to overcome the global impact of neglected tropical diseases: first WHO report on neglected tropical diseases. 2010; 172.

3. Hotez PJ. Neglected infections of poverty in the United States of America. PLoS Negl Trop Dis. 2008; 2: e256. doi:10.1371/journal. pntd.0000256.

4. Hotez PJ, Kamath A. Neglected tropical diseases in sub-Saharan Africa: review of their prevalence, distribution and disease burden. PLoS Negl Trop Dis. 2009; 3(8): e412. doi:10.1371/journal.pntd.0000412.

5. Hotez PJ, Ehrenberg J. Escalating the global fight against neglected tropical diseases through interventions in the Asia Pacific Region. Adv Parasitol. 2010; 72: 31-53. doi: 10.1016/S0065-308X(10)72002-9.

6. Hotez PJ, Woc-Colburn L, Bottazzi ME. Neglected tropical Diseases in Central America and Panama: review of their prevalence, populations at risk and impact on regional development. Int J Parasitol. 2014; 44(9): 597-603. doi: 10.1016/j.ijpara.2014.04.001.

7. Kashino W, Piyaphanee W, Kittitrakul C, Tangpukdee N, Sibunruang S, Lawpoolsri S, et al. Incidence of potential rabies Exposure Among Japanese Expatriates and Travelers in Thailand. J Travel Med. 2014; 21(4): 240-247. doi: 10.1111/jtm.12124.

8. Kappagoda S, Ioannidis JP. Prevention and control of neglected tropical diseases: overview of randomized trials, systematic reviews and meta-analyses. Bull World Health Organ. 2014; 1; 92(5):356366C. doi: 10.2471/BLT.13.129601.

9. Hotez PJ, Savioli L, Fenwick A. Neglected tropical diseases of the Middle East and North Africa: review of their prevalence, distribution, and opportunities for control. PLoS Negl Trop Dis. 2012; 6(2): e1475. doi:10.1371/ journal.pntd.0001475.

10. Helmy MM, Al Mathal IM. Human infection with Onchocerca volvulus in Asir District (Saudi Arabia). J Egypt Soc Parasitol. 2003; 33(2): 385390.
11. Siddiqui MA, al-Khawajah MM. The black disease of Arabia, Sowdaonchocerciasis. New findings.Int J Dermatol. 1991; (2):130-133.

12. Abd El-Salam NM, Ayaz S, Ullah R. PCR and Microscopic Identification of Isolated Leishmania tropica from Clinical Samples of Cutaneous Leishmaniasis in Human Population of Kohat Region in Khyber Pakhtunkhwa. Biomed Res Int. 2014; 2014: 861831. doi. org/10.1155/2014/861831.

13. Jaber SM, Ibbini JH, Hijjawi NS, Amdar NM, Huwail MJ, Al-Aboud K. Exploring recent spatial patterns of cutaneous leishmaniasis and their associations with climate in some countries of the Middle East using geographical information systems. Geospat Health. 2013; 8(1):143158.

14. Almushait MA, Dajem SM, Elsherbiny NM. Seroprevalence and risk factors of Toxoplasma gondii infection among pregnant women in south western, Saudi Arabia. J Parasit Dis. 2014; 38(1): 4-10. doi: 10.1007/s12639-012-0195-z.

15. Alqahtani J, Hassan MM. Incidence of Toxoplasmosis gondii in Najran region, KSA. J Egypt Soc Parasitol. 2012; 42(2): 253-60.

16. Bin Dajem SM, Almushait MA. Detection of Toxoplasma gondii DNA by PCR in blood samples collected from pregnant Saudi women from the Aseer region, Saudi Arabia. Ann Saudi Med. 2012; 32(5): 507-512.

17. Makki SM, Abdel-Tawab AH. Anti-Toxoplasma gondii antibodies among volunteer blood donors in eastern Saudi Arabia. J Egypt Soc Parasitol. 2010; 40(2): 401-412.

18. Alanazi AD. Determination of seropositivity for Toxoplasma gondii in sheep, goats and camels slaughtered for food and human consumptions in Riyadh municipal abattoirs, Saudi Arabia. J Egypt Soc Parasitol. 2013; 43(3): 569-576.

19. Flegr J, Prandota J, Sovic kova' M, Israili ZH. Toxoplasmosis--a global threat. Correlation of latent toxoplasmosis with specific disease burden in aset of 88 countries. PLoS ONE. 2014; 9(3): e90203. doi:10.1371/journal.pone.0090203.

20. Kamal IH, Al Gashgari B, Moselhy SS, Kumosani TA, Abulnaja KO, et al. Two-stage PCR assay for detection of human brucellosis in endemic areas. BMC Infect Dis. 2013; 13: 145. doi: 10.1186/1471-2334-13145 .

21. Asaad AM, Alqahtani JM. Serological and molecular diagnosis of human brucellosis in Najran, Southwestern Saudi Arabia. J Infect Public Health. 2012; 5(2):189-94. doi: 10.1016/j.jiph.2012.02.001.

22. Al-Azraqi TA, El Mekki AA, Mahfouz AA. Seroprevalence of dengue virus infection in Aseer and Jizan regions, Southwestern Saudi Arabia. Trans R Soc Trop Med Hyg. 2013; 107(6): 368-371. doi: 10.1093/ trstmh/trt022.

23. Amarasinghe A, Letson GW. Dengue in the Middle East: a neglected, emerging disease of importance. Trans R Soc Trop Med Hyg. 2012; 106(1):1-2. doi: 10.1016/j.trstmh.2011.08.014.

24. Madani TA, Abuelzein el-TM, Al-Bar HM, Azhar EI, Kao M, Alshoeb HO, et al. Outbreak of viral hemorrhagic fever caused by dengue virus type 3 in Al-Mukalla, Yemen. BMC Infect Dis. 2013; 14; 13:136. doi: 10.1186/1471-2334-13-136.

25. Hassan OA, Ahlm C, Evander M. A need for One Health approachlessons learned from outbreaks of Rift Valley fever in Saudi Arabia and Sudan. Infect Ecol Epidemiol. 2014; 4; 4. doi: 10.3402/iee.v4.20710.

26. Al-Azraqi TA, El Mekki AA, Mahfouz AA. Rift Valley Fever in Southwestern Saudi Arabia: a sero-epidemiological study seven years after the outbreak of 2000-2001. Acta Trop. 2012; 123(2):111-6. doi10.1016/j.actatropica.2012.04.007. 
27. Al-Afaleq AI, Hussein MF. The status of Rift Valley fever in animals in Saudi Arabia: a mini review. Vector Borne Zoonotic Dis 11. 2011; 11(12): 1513-1520. doi: 10.1089/vbz.2010.0245.

28.Zaki AM. Isolation of a flavivirus related to the tick-borne encephalitis complex from human cases in Saudi Arabia. Trans R Soc Trop Med Hyg. 1997; 91:179-181.
29. Memish ZA, Fagbo SF, Osman Ali A, et al. Is the Epidemiology of Alkhurma Hemorrhagic Fever Changing? : A Three-Year Overview in Saudi Arabia. PLoS ONE. 2014; 9(2): e85564. doi:10.1371/journal. pone.0085564.

30. Al-Khalifa MS, Hussein HS, Diab FM, Khalil GM. Blood parasites of livestock in certain Regions in Saudi Arabia. Saudi J Biol Sci. 2009; 16(12): 63-67. doi: 10.1016/j.sjbs.2009.10.002. 